PROCEEDINGS OF THE

AMERICAN MATHEMATICAL SOCIETY

Volume 138, Number 6, June 2010, Pages 1979-1982

S 0002-9939(10)10236-6

Article electronically published on January 13, 2010

\title{
ASYMPTOTIC BEHAVIOUR OF STANDARD BASES
}

\author{
GUILLAUME ROND
}

(Communicated by Bernd Ulrich)

\begin{abstract}
We prove that the elements of any standard basis of $I^{n}$, where $I$ is an ideal of a Noetherian local ring and $n$ is a positive integer, have order bounded by a linear function in $n$. We deduce from this that the elements of any standard basis of $I^{n}$ in the sense of Grauert-Hironaka, where $I$ is an ideal of the ring of power series, have order bounded by a polynomial function in $n$.
\end{abstract}

The aim of this paper is to study the growth of the orders of the elements of a standard basis of $I^{n}$, where $I$ is an ideal of a Noetherian local ring. Here we show that the maximal order of an element of a standard basis of $I^{n}$ is bounded by a linear function in $n$. For this we prove a linear version of the strong Artin-Rees lemma for ideals in a Noetherian ring. The main result of this paper is Theorem 3 .

First we prove the following proposition inspired by Corollary 3.3 of 4 :

Proposition 1. Let $A$ be a Noetherian ring and let $I$ and $J$ be ideals of $A$. There exists an integer $\lambda \geq 0$ such that

$\forall x \in A, \forall n, m \in \mathbb{N}, n \geq \lambda m, \quad\left(x^{n}\right) \cap\left(J+I^{m}\right)=\left(\left(x^{\lambda m}\right) \cap\left(J+I^{m}\right)\right)\left(x^{n-\lambda m}\right)$.

Proof. Let $B:=A / J$. By Theorem 3.4 of [5], there exists $\lambda$ such that for any $m \geq 1$, there exists an irredundant primary decomposition $I^{m}=Q_{1}^{(m)} \cap \cdots \cap Q_{r}^{(m)}$ such that if $P_{i}^{(m)}:=\sqrt{Q_{i}^{(m)}}$, then $\left(P_{i}^{(m)}\right)^{\lambda m} \subset Q_{i}^{(m)}$ for $1 \leq i \leq m$. We denote by $\bar{Q}_{i}^{(m)}$ the image of $Q_{i}^{(m)}$ in $A /\left(J+I^{m}\right)$ for $1 \leq i \leq r$. We denote by $\mathfrak{P}_{i}^{(m)}$ the inverse image of $P_{i}^{(m)}$ in $A$, for $1 \leq i \leq r$.

Let $x \in A$. If $x \in \mathfrak{P}_{i}^{(m)}$, then $x^{n} \in\left(\mathfrak{P}_{i}^{(m)}\right)^{n}$ and $\left({\overline{Q_{i}}}^{(m)}: x^{n}\right)=A /\left(J+I^{m}\right)$ for any $n \geq \lambda m$. If $x \notin \mathfrak{P}_{i}^{(m)}$, then $x^{n} \notin\left(\mathfrak{P}_{i}^{(m)}\right)^{n}$ and $\left(\bar{Q}_{i}^{(m)}: x^{n}\right)=\bar{Q}_{i}^{(m)}$ for any $n \geq \lambda m$. Thus, for any $n \geq \lambda m$,

$$
\left(0_{A /\left(J+I^{m}\right)}: x^{n}\right)=\left(\bigcap_{i} \bar{Q}_{i}^{(m)}: x^{n}\right)=\bigcap_{i}\left(\bar{Q}_{i}^{(m)}: x^{n}\right)=\bigcap_{i / x \notin P_{i}} \bar{Q}_{i}^{(m)}
$$

Hence, by Remark 2 (1) of [4] and Theorem 2 of [4], we get the result.

Using the extended Rees algebra of $\mathfrak{a}$ to reduce to the principal case (as done in [5]), we prove the following corollary:

Received by the editors January 21, 2009, and, in revised form, October 1, 2009. 2010 Mathematics Subject Classification. Primary 13H99, 13 C99.

(C)2010 American Mathematical Society Reverts to public domain 28 years from publication 
Corollary 2. Let $A$ be a Noetherian ring and let $I, J$ and $\mathfrak{a}$ be ideals of $A$. Then there exists $\lambda \geq 0$ such that

$$
\left(J+I^{m}\right) \cap \mathfrak{a}^{n}=\left(\left(J+I^{m}\right) \cap \mathfrak{a}^{\lambda m}\right) \mathfrak{a}^{n-\lambda m} .
$$

Proof. Let $B:=A\left[\mathfrak{a} t, t^{-1}\right]$. Then $t^{-n} B \cap A=\mathfrak{a}^{n}$. By Proposition 1, there exists $\lambda \geq 1$ such that for any $n, m \in \mathbb{N}, n \geq \lambda m$,

$$
\left(t^{-n}\right) \cap\left(J+I^{m}\right)=\left(\left(t^{-\lambda m}\right) \cap\left(J+I^{m}\right)\right)\left(t^{-(n-\lambda m)}\right) .
$$

We have

$$
\left(J+I^{m}\right) \cap \mathfrak{a}^{n}=\left(\left(t^{-m}\right) \cap\left(J+I^{m}\right) B\right) \cap A=\left(\left(\left(t^{-\lambda m}\right) \cap\left(J+I^{m}\right)\right)\left(t^{-(n-\lambda m)}\right)\right) \cap A .
$$

Thus $\left(J+I^{m}\right) \cap \mathfrak{a}^{n} \subset\left(\left(J+I^{m}\right) \cap \mathfrak{a}^{\lambda m}\right) \mathfrak{a}^{n-\lambda m}$. The reverse inclusion is clear.

Let $(A, \mathfrak{m})$ be a Noetherian local ring and $I$ be an ideal of $A$. Let us denote by $G(A / I)$ the associated graded ring of $A / I$ with respect to $\mathfrak{m}$. Then $G(A / I)=$ $G(A) / I^{*}$, where $I^{*} \subset G(A)$ is the graded ideal of $G(A)$ generated by the elements $f^{*}$ with $f \in I$, where $f^{*}$ is the leading form of $f:$ if $\operatorname{ord}(f):=\sup \left\{k, f \in \mathfrak{m}^{k}\right\}=d$, then $f^{*}=f+\mathfrak{m}^{d+1}$. Finally $f_{1}, \ldots, f_{p}$ form a (minimal) standard basis of $I$ if $f_{1}^{*}, \ldots, f_{p}^{*}$ form a (minimal) generating set of $I^{*}$. It is clear that $\left(I^{*}\right)^{n}$ is included in $\left(I^{n}\right)^{*}$, but both ideals are not equal in general. For example, if $I=\left(x^{2}, y^{3}-x y\right) \subset$ $\mathbb{k}[[x, y]]$ where $\mathbb{k}$ is a field, then $\left(I^{n}\right)^{*}=\left(\left(x y, x^{2}\right)^{n},\left\{x^{i} y^{4 n-3 i+1}\right\}_{0 \leq i \leq n-1}\right)$; hence $y^{4 n+1} \in\left(I^{n}\right)^{*} \backslash\left(I^{*}\right)^{n}$ 2. Nevertheless we have the following theorem whose proof is inspired by the link made in [1] between the Artin-Rees lemma and the orders of the elements of a standard basis, with respect to a monomial order, of an ideal in the ring of formal power series over a field (see also [6]).

Theorem 3. Let $I$ be an ideal of a Noetherian local ring $(A, \mathfrak{m})$. Then there exists an integer $\lambda \geq 0$ such that for any integer $n \geq 0$ and any minimal standard basis $f_{1}, \ldots, f_{p_{n}}$ of $I^{n}$ we have ord $\left(f_{i}\right) \leq \lambda n$ for $1 \leq i \leq p_{n}$.

Proof. The canonical morphism $A \longrightarrow \widehat{A}$ is injective and $G(A / I)=G(\widehat{A / I})$. Thus we may assume that $A$ is complete. Then $A$ is of the form $B / J$, where $B$ is a regular local ring and $J$ is an ideal of $B$. Hence we may assume that $A$ is a regular local ring, $I$ and $J$ are ideals of $A$, and we need to prove that there exists $\lambda \geq 0$ such that for any minimal standard basis $f_{1}, \ldots, f_{p_{n}}$ of $J+I^{n}$ we have $\operatorname{ord}\left(f_{i}\right) \leq \lambda n$ for $1 \leq i \leq p_{n}$.

Let us assume that $I+J \neq(0)$. Let $n \in \mathbb{N}^{*}$ and let $f_{1}, \ldots, f_{p_{n}} \in J+I^{n}$ such that $f_{1}^{*}, \ldots, f_{p_{n}}^{*}$ form a minimal generating set of $\left(J+I^{n}\right)^{*}$ (in particular, $\left.\left(f_{1}, \ldots, f_{p_{n}}\right)=J+I^{n}\right)$. Let us denote by $r_{i}$ the integer $\operatorname{ord}\left(f_{i}\right), 1 \leq i \leq p_{n}$, and let us assume that $r_{1} \leq r_{2} \leq \cdots \leq r_{p_{n}}$. Let $\lambda \geq 0$ satisfy Corollary 2 with $\mathfrak{a}=\mathfrak{m}$. Let $q \geq 0$ such that $r_{i} \leq \lambda n$ for $i \leq q$ and $r_{i}>\lambda n$ for $i>q$. It is enough to show that $q=p_{n}$. Let us assume that $q<p_{n}$. If $q=0$, then $f_{i} \in\left(J+I^{n}\right) \cap \mathfrak{m}^{r_{i}}=\left(\left(J+I^{n}\right) \cap \mathfrak{m}^{\lambda n}\right) \mathfrak{m}^{r_{i}-\lambda n} \subset\left(J+I^{n}\right) \mathfrak{m}, 1 \leq i \leq p_{n}$. Hence $\left(J+I^{n}\right)=\mathfrak{m}\left(J+I^{n}\right)$, and $\left(J+I^{n}\right)=(0)$ by Nakayama, which is a contradiction. Thus $q \geq 1$. For $i>q$ we have $f_{i} \in\left(J+I^{n}\right) \cap \mathfrak{m}^{r_{i}}=\left(\left(J+I^{n}\right) \cap \mathfrak{m}^{\lambda n}\right) \mathfrak{m}^{r_{i}-\lambda n}$. Thus, for $q+1 \leq i \leq p_{n}, f_{i}=\sum_{k} \varepsilon_{i, k} g_{i, k}$ with $g_{i, k} \in\left(J+I^{n}\right) \cap \mathfrak{m}^{\lambda n} \varepsilon_{i, k} \in \mathfrak{m}^{r_{i}-\lambda n}$ for $q<i \leq p_{n}$ and any $k$. Hence $f_{i}=\sum_{k} \varepsilon_{i, k}\left(\sum_{1 \leq l \leq p_{n}} \eta_{i, k, l} f_{l}\right)$ with $\eta_{i, k, l} \in \mathfrak{m}^{\lambda n-r_{l}}$ for any $i, k, l$ (because $f_{1}^{*}, \ldots, f_{p_{n}}^{*}$ generate $\left(J+I^{n}\right)^{*}$ and $G(A)$ is an integral domain). 
Thus, for $q<i \leq p_{n}$,

$$
f_{i}=\left(1-\sum_{k} \varepsilon_{i, k} \eta_{i, k, i}\right)^{-1} \sum_{k} \varepsilon_{i, k}\left(\sum_{l \neq i} \eta_{i, k, l} f_{l}\right) .
$$

Then $f_{i} \in \sum_{l \neq i} f_{l} \mathfrak{m}^{r_{i}-r_{l}}$ for $q+1 \leq i \leq p_{n}$. By Gaussian elimination we see that

$$
f_{i} \in \sum_{l<i} f_{l} \mathfrak{m}^{r_{i}-r_{l}} \text { for } q+1 \leq i \leq p_{n} .
$$

This means that $f_{i}^{*} \in\left(f_{1}^{*}, \ldots, f_{i-1}^{*}\right) G(A)$, which contradicts the fact that $f_{1}^{*}, \ldots, f_{p_{n}}^{*}$ form a minimal generating set of $\left(J+I^{n}\right)^{*}$.

Let $\mathcal{O}_{s}:=\mathbb{k}\left[\left[x_{1}, \ldots, x_{s}\right]\right]$, where $\mathbb{k}$ is a field, or let $\mathcal{O}_{s}:=\mathbb{k}\left\{x_{1}, \ldots, x_{s}\right\}$, where $\mathbb{k}$ is a valued field. We denote by $\mathfrak{m}$ its maximal ideal. For all $\alpha \in \mathbb{N}^{s}$ let us denote $|\alpha|:=\alpha_{1}+\cdots+\alpha_{s}$. We define a total order on $\mathbb{N}^{s}$ in the following way: $\alpha>\beta$ if $\left(|\alpha|, \alpha_{1}, \ldots, \alpha_{s}\right)>_{\text {lex }}\left(|\beta|, \beta_{1}, \ldots, \beta_{s}\right)$ for all $\alpha, \beta \in \mathbb{N}^{s}$. This induces a total order on the monomials of $\mathcal{O}_{s}$ in the following way: $x^{\alpha}>x^{\beta}$ if $\alpha>\beta$ for all $\alpha, \beta \in \mathbb{N}^{s}$. If $f=\sum_{\alpha \in \mathbb{N}^{s}} f_{\alpha} x^{\alpha} \in \mathcal{O}_{s}$, let us denote by $\operatorname{in}_{>}(f)$ the element $f_{\alpha} x^{\alpha}$ such that $\alpha<\beta$ for all $\beta \neq \alpha$ such that $f_{\beta} \neq 0$. If $\operatorname{in}_{>}(f)=f_{\alpha} x^{\alpha}$, let us denote by $\exp (f)$ the element $\alpha \in \mathbb{N}^{s}$. Let $I$ be an ideal of $\mathcal{O}_{s}$; we say that $\left(f_{1}, \ldots, f_{p}\right)$ is a (minimal) standard basis of I with respect to this order if $\left\{\exp \left(f_{1}\right), \ldots, \exp \left(f_{p}\right)\right\}$ is a (minimal) set of generators of the semigroup $\{\exp (g), g \in I\}$ (in particular $\left(f_{1}, \ldots, f_{p}\right)=I$ ). We denote $\alpha_{i}:=\exp \left(f_{i}\right)$ for all $i$. We may always assume that $\left|\alpha_{1}\right| \leq \cdots \leq\left|\alpha_{p}\right|$. In this case, for $l \in \mathbb{N}$ we define $q(l) \in \mathbb{N}$ by $\alpha_{q(l)} \leq l$ and $\alpha_{q(l)+1}>l$, where $q(l)=0$ if $l<\left|\alpha_{1}\right|$ and $q(l)=p$ if $l \geq\left|\alpha_{p}\right|$. We have the following result:

Proposition 4 ([6]). Let $I$ be and ideal of $\mathcal{O}_{s}$. Then, with the previous notation,

$$
I \cap \mathfrak{m}^{m+l}=\left(I \cap \mathfrak{m}^{l}\right) \mathfrak{m}^{m} \text { for all } m \geq 0
$$

if and only if $r(l) \geq 1$ and $f_{j} \in \mathfrak{m}^{\left|\alpha_{j}\right|-\left|\alpha_{1}\right|} f_{1}+\cdots+\mathfrak{m}^{\left|\alpha_{j}\right|-\left|\alpha_{r(l)}\right|} f_{r(l)}$, for $j=$ $r(l)+1, \ldots, p$.

Corollary 5. Let $I$ be an ideal of $\mathcal{O}_{s}$. Then there exists a polynomial function in $n$, denoted by $P$, such that for all integers $n \geq 0$ and any minimal standard basis $f_{1}, \ldots, f_{p_{n}}$ of $I^{n}$ with respect to in $n_{>}$we have $\operatorname{ord}\left(f_{i}\right) \leq P(n)$ for $1 \leq i \leq p_{n}$.

Proof. Let $f_{1}, \ldots, f_{p_{n}}$ be a minimal standard basis of $I^{n}$ with respect to $i_{>}$. Let $\alpha_{i}:=\exp \left(f_{i}\right), 1 \leq i \leq p_{n}$, and let us assume that $\alpha_{1} \leq \alpha_{2} \leq \cdots \leq \alpha_{p_{n}}$. The sequence $\alpha_{1}, \ldots, \alpha_{p_{n}}$ is uniquely determined by $I^{n}$. By applying Proposition 4 and Corollary 2, we see that there exists $\lambda \geq 0$, not depending on $n$, such that $\left|\alpha_{1}\right| \leq\left|\alpha_{2}\right| \leq \cdots \leq\left|\alpha_{r}\right| \leq \lambda n<\left|\alpha_{r+1}\right| \leq \cdots \leq\left|\alpha_{p_{n}}\right|$ and

$$
f_{i} \in \mathfrak{m}^{\left|\alpha_{i}\right|-\left|\alpha_{1}\right|} f_{1}+\cdots+\mathfrak{m}^{\left|\alpha_{i}\right|-\left|\alpha_{r}\right|} f_{r} \quad \text { for } r+1 \leq i \leq p_{n} .
$$

In particular, $\left(f_{1}^{*}, \ldots, f_{r}^{*}\right)$ is a system of generators of $\left(I^{n}\right)^{*}$, and $\left(f_{1}^{*}, \ldots, f_{p_{n}}^{*}\right)$ is a Gröbner basis of the homogeneous ideal $\left(I^{n}\right)^{*}$ with respect to the graded lexicographic order. From [3] $\operatorname{ord}\left(f_{i}^{*}\right)$ is bounded by a polynomial function in $\lambda n$ depending only on $I$ and $s$, for $r+1 \leq i \leq p_{n}$. This proves the corollary.

\section{ACKNOWLEDGMENT}

The author would like to thank Irena Swanson for having taken the time to answer his many questions about the subject. 


\section{REFERENCES}

1. E. Bierstone, P. Milman, The local geometry of analytic mappings, Universita di Pisa, ETS Editrice, Pisa, 1988. MR971251 (90j:32011)

2. S. D. Cutkosky, J. Herzog, H. Srinivasan, Finite Generation of Algebras Associated to Powers of Ideals, arXiv 0806.0566, preprint.

3. H. Möller, F. Mora, Upper and lower bounds for the degree of Groebner bases, EUROSAM 84 (Cambridge, 1984), 172-183, Lecture Notes in Comput. Sci., 174, Springer, Berlin, 1984. MR:779124 (86k:13008)

4. F. Planas-Vilanova, The strong uniform Artin-Rees property in codimension one, J. Reine Angew. Math., 527 (2000), 185-201. MR1794022(2001g:13051)

5. I. Swanson, Powers of ideals. Primary decompositions, Artin-Rees lemma and regularity, Math. Ann., 307 (1997), 299-313. MR.1428875 (97j:13005)

6. T. Wang, A stratification given by Artin-Rees estimates, Can. J. Math., 44 (1) (1992), 194205. MR.1152675 (93e:13031)

Institut de Mathématiques de Luminy, Université Aix-Marseille 2, Campus de Luminy, CaSe 907, 13288 Marseille Cedex 9, France

E-mail address: rond@iml.univ-mrs.fr 